This manuscript has been authored by UT-Battelle, LLC under Contract No. DE-AC05-00OR22725 with the U.S. Department of Energy. The United States Government retains and the publisher, by accepting the article for publication, acknowledges that the United States Government retains a non-exclusive, paid-up, irrevocable, world-wide license to publish or reproduce the published form of this manuscript, or allow others to do so, for United States Government purposes. The Department of Energy will provide public access to these results of federally sponsored research in accordance with the DOE Public Access Plan (http://energy.gov/downloads/doe-public-access-plan). 


\title{
Effects of geometric factors and shear band patterns on notch sensitivity in bulk metallic glasses
}

\author{
Weidong Li, ${ }^{1}$ Hongbin Bei, ${ }^{2, *}$ Yanfei Gao ${ }^{1,2, *}$ \\ ${ }^{1}$ Department of Materials Science and Engineering, University of Tennessee, Knoxville, TN 37996 \\ ${ }^{2}$ Materials Science and Technology Division, Oak Ridge National Laboratory, Oak Ridge, TN 37831
}

\begin{abstract}
Recent experiments in notched bulk metallic glasses have found reduced, or insensitive, or improved strengths, while in many of these cases the ductile strain prior to final failure is enhanced. First, although the inverse notch effect is explained by a shift from shear localization to cavitation failure, it is suggested in this work that the synergistic effect between cohesive fracture at the notched area and shear bands emanating from the notch roots may extend the parametric space for the notch insensitive behavior. Second, the dependence of shear band patterns on notch geometric factors is determined by the Rudnicki-Rice theory and the freevolume-based finite element simulations. These results suggest conditions for shear band multiplication to take place and for the shear-localization-induced failure to be delayed.
\end{abstract}

Keywords: notch sensitivity; cohesive crack; shear bands

*Corresponding information: beih@ornl.gov; ygao7@utk.edu 


\section{Introduction}

In contrast to crystalline metals, bulk metallic glasses (BMGs) possess amorphous packing of the constituent metallic elements, and thus do not involve plasticity mechanisms arising from dislocations and grain boundaries. At temperatures lower than the glass transition temperature, BMGs deform by strain localization into narrow shear bands, which rapidly lead to catastrophic failure if these shear bands are not geometrically blocked. Consequently, BMGs are oftentimes brittle, and the lack of ductility prior to fracture failure prevents their wide applications as engineering structural materials.

The study of notches, cracks, and more generally flaws is of paramount importance in engineering applications, because failures are likely to take place at these geometric irregularities and stress risers. It appears from the linear elastic fracture mechanics (LEFM) that notches and flaws will reduce the strength of BMGs as in other brittle solids. However, recent studies have reported a diverse variety of observations on notched BMGs [1-8]. As summarized in Pan et al. [7] and shown in the geometric setup in Fig. 1(a), the notch shape can be described by a geometric ratio, $\lambda=(L-2 a) / w$. With the increase of $\lambda$, Flores and Dauskardt [1] found the reduction of the failure stress for notched cylindrical samples, termed as positive notch effect; Qu et al. [4] found notch sensitivity of the failure stress for notched plate samples; and Pan et al. [7] found the increase of the failure stress for notched cylindrical samples, termed as inverse notch effect. These results are rationalized in Pan et al. [7] by the competition between cavitation failure at the center of the notched area and shear bands adjacent to the notch roots. As these two mechanisms are dictated by the hydrostatic stress, $\sigma_{m}$, and Mises stress, $\sigma_{e q}$, respectively, their ratio for a notched sample is given by

$$
\frac{\sigma_{m}}{\sigma_{e q}}=\frac{1}{3}+\ln \left(1+\frac{\lambda}{2}\right) .
$$

Then they introduced a material parameter, $\sigma_{c} / \sigma_{s}$, where $\sigma_{c}$ and $\sigma_{s}$ are the critical stresses for cavitation and shear localization, respectively. If $\sigma_{c} / \sigma_{s}$ is very large (e.g., >2), the increase of $\lambda$ will never result into a hydrostatic stress that reaches $\sigma_{c}$, since the shear localization condition will be first reached. This corresponds to the positive notch effect. For a small $\sigma_{c} / \sigma_{s}$ (e.g., 1.4 in Pan et al. [7]), the increase of $\lambda$ leads to a transition from shear localization to cavitation failure, corresponding to the inverse notch effect. 
One important experimental observation that may not be rationalized by the above model is the size effect or flaw insensitivity at small dimensions in BMGs [9-11]. Consider a central crack with the length of $2 a$ subjected to a faraway tensile stress of $\sigma_{a p p l}$. The LEFM analysis predicts that the tensile strength scales inversely to the square root of the crack size by

$$
\sigma_{c r t}=K_{I c} / \sqrt{\pi a} \text {. }
$$

Stress singularity cannot be reached in reality, and the flaw insensitivity observed in small dimensions apparently violates this prediction. Prior efforts in nonlinear fracture mechanics and composites have revealed the critical role played by the inelastic process zone in interpreting the observation of flaw insensitivity. For an example, the plastic zone near the crack tip has a size of $r_{p} \approx \frac{1}{3 \pi}\left(\frac{K_{a p p l}}{\sigma_{Y}}\right)^{2}$ with the yield strength $\sigma_{Y}$ and the applied stress intensity factor $K_{a p p l}$. The LEFM analysis is limited to the condition of $r_{p}<<a$. When $r_{p}$ is comparable to the crack size, the nonlinear fracture mechanics should be employed. When $r_{p}$ is larger than the crack, a stressbased ductile failure criterion, such as the Gurson-Tvergaard model, should be used [12]. In the last case, the scaling in Eq. (2) is invalid and these flaws, since they are smaller than the plastic zone, will not reduce the tensile strength noticeably. For the other example, the inelastic process zone can be a bridging zone arising from atomic cohesion in brittle solids or fiber pull-out in composites [13-15]. As shown in Fig. 2, the singular stress field predicted by LEFM is now replaced by the solution from a cohesive interface in the crack advancing direction. A representative cohesive law is given in Fig. 2(b) with the interface strength $\sigma_{0}$ and the characteristic length $\delta_{0}$. The flaw insensitivity is thus dictated by the comparison between the crack size and the cohesive zone size, given by $E \delta_{0} / \sigma_{0}$. For brittle solids, $\sigma_{0}$ and $\delta_{0}$ derive from interatomic interactions, and the ratio of $E \delta_{0} / \sigma_{0}$ is on the order of nanometer. Therefore, except for vacancies or small vacancy clusters, flaws are usually detrimental in brittle solids. The classic Dugdale strip-yield model is mathematically equivalent to this cohesive interface model, so that the Dugdale plastic zone size can also be given by the above length when $\sigma_{0}$ is interpreted as yield stress. For fiber-reinforced composites, $\sigma_{0}$ and $\delta_{0}$ are governed by the fiber pull-out process and the fiber volume fraction, and the cohesive zone size can extend to millimeters. In this case, microscopic flaws have virtually no effects on the composite strength. 
For a third example, we note that the long-chain molecular bonds in biological materials govern the interface adhesion [15]. For applications like cell adhesion, the cohesive zone size can indeed be very large, so the use of LEFM should be cautious.

The concept of crack bridging can certainly be applied to the BMG study, where the cohesive interface law in Fig. 2(b) can be used as a simplified description of the normal fracture process. Molecular simulations of small-sized BMGs in $[9,10]$ resemble the large-scale-bridging characteristics (i.e., $E \delta_{0} / \sigma_{0}>>a$ ), so that the failure strength is not governed by the flaw size as predicted by the LEFM. In addition, He et al. [14] found out that a localized band with reduced strength or stiffness, if emanating from a crack tip, will relax the stress concentration and effectively extend the cohesive zone length. It is thus anticipated that the synergy between the cohesive fracture and shear localization can extend the parametric space for the notchinsensitivity behavior. Along this line, the proposed governing boundary value problem in Fig. 2 will be investigated in Section 2 of this work.

The other experimental observation that cannot be directly explained by the cavitation versus shear localization model is the ductility enhancement in notched BMGs [2-5,7]. It is well known that shear bands will propagate catastrophically in unconstrained samples and little ductility is thus observed. To this end, the most efficient method in ductility improvement is the introduction of localized stress fields or geometric features that prevent the shear band running through the entire sample, and also promote the multiplication of shear bands [16-18]. Notched BMGs certainly have stress concentrations confined near notch roots for large $\lambda$, where many short shear bands will be expected to appear. With the decrease of $\lambda$, not only the ratio of $\sigma_{m} / \sigma_{e q}$ decreases so that cavitation failure becomes unlikely, but also the geometric constraint to the shear band propagation is reduced. In this case, the notched BMGs behave as long-andthin, pillar-like samples sandwiched between two loading platens, and therefore the unconstrained shear band propagation will govern the failure. Most previous simulations either do not directly simulate the individual shear bands (rather, continuum plasticity model is often employed [7,19-21]), or are restricted in computational capabilities (e.g., molecular simulations). In this work, the instability theories and free-volume-based finite element method are used to systematically investigate the relationship between shear bands and geometric factors of notched BMGs, as will be presented in Section 3. 


\section{Notch sensitivity of strength: cohesive fracture and shear localization}

In this section, the synergy between the normal fracture along the notched plane and the shear band in an inclined direction to the loading direction is simulated by the cohesive interface model. As addressed in Section 1, this is the first line that complements to the model in Eq. (1). The geometric setup of a double-edge notch/crack sample is presented in Fig. 2(a), with only a quarter plane illustrated due to the symmetry argument. The notched zone is represented by a cohesive interface model, with the normal traction, $T_{n}$, and the normal separation, $\Delta_{n}$, related by

$$
\frac{T_{n}}{\sigma_{0}}=\frac{\Delta_{n}}{\delta_{0}} \exp \left(1-\frac{\Delta_{n}}{\delta_{0}}\right)
$$

as depicted in Fig. 2(b). For the Mode-I loading condition, there is no need to specify the shear response of the cohesive interface. The cohesive elements are implemented into the commercial finite element software, ABAQUS, through the user-defined element (UEL) subroutine [22]. From a top-down approach, the cohesive interface model allows us to separately investigate the contribution of the inelastic process zone to the crack nucleation and growth resistance [23-25]. For example, Tvergaard and Hutchinson [23] determined the crack growth resistance curve due to the plastic deformation in the vicinity of the crack tip. In notched BMGs, the inelastic process zone consists of shear bands from the crack tip. Following He et al. [14], we represent the shear band by a weak zone with the shear traction, $T_{t}$, and the shear separation, $\Delta_{t}$, given in Fig. 2(c),

$$
\frac{T_{t}}{\tau_{s}}= \begin{cases}\Delta_{t} / \delta_{s}, & \left|\Delta_{t}\right| \leq \delta_{s} \\ \operatorname{sgn}\left(\Delta_{t}\right), & \left|\Delta_{t}\right|>\delta_{s}\end{cases}
$$

The normal response is assumed to be infinitely stiff. This model is implemented as a different UEL subroutine with interface elements along the weak zone in Fig. 2(a). The inclined angle is taken to be $41^{\circ}$ from the free-volume-based simulations near the notch root to be presented in next section. This angle is different from the inclined angles in uniaxial tests $[16,26]$. The extent of the weak zone is determined from the boundary value problem. That is, from the crack tip along the shear band direction, the shear stress decreases rapidly to zero. The red zone in Fig. 2(a) corresponds to the regime where the shear stress reaches $\tau_{s}$ and thus denotes the extent of the shear band.

It should be noted that the above two types of cohesive zone models treat normal and shear responses separately. For metallic glasses, a shear band can certainly evolve into a crack 
when a large plastic strain can be achieved such as under constrained geometric condition. Recent molecular simulations suggest that this process involve cavitation inside the shear bands [27], which cannot be modeled by the Gurson-Tvergaard model or the simplified model in Eqs. (3) and (4). A thorough treatment along this line is out of the scope of this work; rather, we are concerned with the role of shear bands that delays the cohesive fracture.

In the finite element simulations, the two cohesive planes are modeled by the UEL subroutines as explained above, and the surrounding solid is elastic with the Young's modulus $E$ and Poisson's ratio $v$ (taken as 0.3). With the application of a displacement boundary condition in Fig. 2(a), the resulting tensile stress is recorded and its peak value is the fracture strength. The fracture strength, $\sigma_{c r t}$, as normalized by $\sigma_{0}$ and the geometric factor $L /(L-2 a)$, is given as a function of $a \sigma_{0} / E \delta_{0}$ in Fig. 3. If the ratio of $\tau_{s} / \sigma_{0}$ is large, the weak zone has no effect to the fracture process, as given by the circle-marked curve. When the cohesive zone length is small, i.e., $E \delta_{0} / \sigma_{0}<<a$, the LEFM analysis is valid, so that the prediction in Eq. (2) gives the inverse square-root dependence of $\sigma_{c r t}$ on $a$, as reproduced in the simulations in Fig. 3 at large $a \sigma_{0} / E \delta_{0}$. This is termed the small-scale-bridging (SSB) behavior in [13]. When the cohesive zone length is large, i.e., $E \delta_{0} / \sigma_{0} \sim a$, the fracture strength reaches the interface strength, i.e., $\sigma_{c r t} \rightarrow \sigma_{0}$, as the failure process is governed by the almost uniform separation of the cohesive interface. This is the large-scale-bridging (LSB) behavior in [13]. As explained in Section 1, the LSB behavior can never be reached in brittle solids if the governing mechanism is atomic decohesion, unless the flaw is of atomic size. For nanocrystalline metals, the deformation mechanisms by grain boundary diffusion/sliding, cooperative motion and rotation of grains, and dislocation nucleation and annihilation at the grain boundaries may render a large cohesive zone size.

As suggested by He et al. [14], the most effective way to broaden the notch-insensitivity regime is by the introduction of weak zones or shear bands at the notch root or crack tip. As presented in Fig. 3, when the shear strength $\tau_{s}$ is much less than the normal strength $\sigma_{0}$, or the shear stiffness is lower, i.e., $\tau_{s} / \delta_{s}<<\sigma_{0} / \delta_{0}$, the weak zone significantly reduces the stress concentration at the crack tip, and thus the notch insensitivity can be found in a wide regime of $a \sigma_{0} / E \delta_{0}$. In uniaxial tests, if $\tau_{s} / \sigma_{0}>1 / \sqrt{2}$, the tensile cohesive fracture will be unlikely to take 
place [26]. The complex stress states near the notch does not permit such a simply analysis, and also the initial stiffnesses in Figs. 2(b) and 2(c) plays a critical role. In the extreme case of $\tau_{s} / \sigma_{0}=0.1$ and $\delta_{s} / \delta_{0}=10$, results at $a \sigma_{0} / E \delta_{0}>10$ are not trustworthy, because the extent of the weak zone reaches the right boundary in Fig. 2 and the failure will thus be governed by the catastrophic propagation of shear bands. This is a failure mode that should be avoided for the purpose of ductility enhancement.

Referring back to the model in Pan et al. [7], the above results in Figs. 2 and 3 suggest that the transition from cavitation failure to shear localization is not a sharp transition. The synergistic effects of shear bands near the notch roots and cavitation failure along the notched area are in fact beneficial in promoting the LSB behavior and thus the notch insensitivity. In the experiment by Jiang et al. [28], the failure mode changes from the shear-localization-induced to the tension-normal failure when the temperature reduces to near $4.2 \mathrm{~K}$ (liquid helium). The latter also exhibits strength scatter, as in typical brittle glasses and ceramics. Such a failure mode change is very likely due to the different dependence of $\tau_{s}$ and $\sigma_{0}$ on the temperature. Lastly, it should be noted that our model in Fig. 2 does not permit the shear-localization-induced failure [29,30]. Results in Fig. 3 rather indicate that the weak zone will delay the normal fracture along the notch plane. An advanced model that considers failure from shear bands will extend the applicability of our model in this work.

\section{Notch sensitivity of ductility: shear band patterns}

The ductility of notched BMGs can be improved if the shear bands can be blocked by geometric constraints or confined by decaying stress fields. Consequently, more shear bands will be initiated, each shear band will experience a lower degree of plastic strain, and thus the transition from shear band to shear crack will be more significantly delayed. Typical shear bands observed in notched BMGs are illustrated in Fig. 4. Radial shear bands in Fig. 4(a), e.g., [2,3], are more commonly observed than the other two sets. The semi-circular or horizontal shear bands in Fig. 4(b), e.g., [4,5], are found only in thin plate samples because these shear bands are caused by out-of-plane shear offsets. When the notch height is larger than notch spacing in Fig. 4(c), a simple geometric argument will suggest an unconstrained propagation of shear band across the notched area. This is another reason for this geometry with low $\lambda$ to be weaker than the geometry with high $\lambda$, in addition to the $\sigma_{c} / \sigma_{s}$ model presented in Pan et al. [7]. 
In this section, our objective is to predict the shear band patterns and to investigate the geometric conditions that favor multiple shear bands and thus reduce the strain on each shear band. In contrast to the introduction of the weak zone in Fig. 2, shear bands will be simulated from two methods: the Rudnicki-Rice instability theory [31] and the free-volume-based constitutive model [32]. In the former, the onset of shear localization corresponds to the loss of ellipticity in the constitutive law. Rudnicki and Rice [31] found that the shear band plane is parallel to the second principal stress, and makes an angle of $\theta_{0}$ with the first principal stress by

$$
\theta_{0}= \pm \tan ^{-1} \sqrt{\frac{\xi-N_{\min }}{N_{\max }-\xi}}
$$

where $\xi=\frac{1}{3}(1+v)(\mu+\beta)-N(1-v)$, Poisson's ratio $v$, the coefficient of the internal friction $\mu$, and the dilatancy factor $\beta$. The principal deviatoric stress components, $\sigma_{I}^{\prime}, \sigma_{I I}^{\prime}$, and $\sigma_{I I I}^{\prime}$, are regrouped to give $N_{\max }=\sqrt{3} \frac{\sigma_{I}^{\prime}}{\sigma_{e q}}, N=\sqrt{3} \frac{\sigma_{I I}^{\prime}}{\sigma_{e q}}$, and $N_{\min }=\sqrt{3} \frac{\sigma_{I I I}^{\prime}}{\sigma_{e q}}$. Example studies in [1618] found little effects of $\mu+\beta$ in the shear band patterns, so that we choose $\mu+\beta$ in this study. We have systematically varied the geometric parameters, $r, a$, and $w$ in Figs. 5-7, and the predictions will be discussed together with the free-volume-based finite element simulation results.

The above Rudnicki-Rice model predicts the possible shear band patterns from the elastic stress field, but it cannot determine the evolution of shear bands and their interactions. Shear bands predicted in Figs. 5-7 are simply discontinued at a certain height. While a number of constitutive laws have been proposed for BMGs, here we adopt the Spaepen-type free volume model [32] and conduct finite element simulations through an ABAQUS user-defined material (UMAT) subroutine [33]. Extending the free volume model in [32] to the multiaxial stress state, the rate of the plastic strain tensor, $\varepsilon_{i j}^{p}$, is given by

$$
\frac{\partial \varepsilon_{i j}^{p}}{\partial t}=f \exp \left(-\frac{\Delta G^{m}}{k_{B} T}\right) \exp \left(-\frac{\alpha v^{*}}{v_{f}}\right) \sinh \left(\frac{\sigma_{e q} \Omega}{2 k_{B} T}\right) \frac{S_{i j}}{\sigma_{e q}}
$$

with $f$ being the frequency of the atomic vibration, $\alpha$ a geometric factor on the order of $1, v^{*}$ the hard-sphere volume of an atom, $v_{f}$ the average free volume per atom, $\Delta G^{m}$ the activation energy, $\Omega$ the atomic volume, $k_{B}$ the Boltzmann constant, and $T$ the absolute temperature. The 
flow direction is proportional to the deviatoric stress tensor, $S_{i j}$. The free volume is treated as an internal variable that describes the structural information. As governed by the competition between a stress-driven disordering process and a diffusional ordering process, its evolution is given by

$$
\frac{\partial v_{f}}{\partial t}=v^{*} f \exp \left(-\frac{\Delta G^{m}}{k_{B} T}\right) \exp \left(-\frac{\alpha v^{*}}{v_{f}}\right)\left\{\frac{2 \alpha k_{B} T}{v_{f} C_{e f f}}\left[\cosh \left(\frac{\sigma_{e q} \Omega}{2 k_{B} T}\right)-1\right]-\frac{1}{n_{D}}\right\},
$$

with $n_{D}$ being the number of atomic jumps needed to annihilate a free volume equal to $v^{*}$, the effective modulus $C_{\text {eff }}=E / 3(1-v)$, and $E$ being the Young's modulus. Example finite element simulations can be found in $[8,17]$. In our simulations, the boundary value problem is given in Fig. 1(a). We choose the following constitutive parameters: $E / \sigma_{r e f}=240, v=0.333, n_{D}=3$, $\alpha=0.15$, and $\sigma_{\text {ref }}=2 k_{B} T / \Omega$. Since the constitutive law does not possess a length scale, the simulated shear band width is limited by the mesh size. Thus our finite element simulations use four-node quadrilateral elements with fine mesh density throughout the entire sample.

Effects of the notch radius are presented in Fig. 5. In the Rudnicki-Rice model, we first calculate the elastic stress field, and then use Eq. (5) to determine the shear band direction at any material point. Streamline plots starting from a number of seed points at the notch roots will give the predicted shear band patterns. The inclined shear bands in Figs. 5(a)-(c) are caused by the inplane shear, while the horizontal shear bands connecting the two notches deform in out-of-plane direction. The change of the notch radius does not affect the inclination angle noticeably. Freevolume-based finite element simulations in Figs. 5(d)-(f) exhibit a much smaller number of shear bands than the predictions from the Rudnicki-Rice model, mainly because the reduction of stress concentration when one or several major shear bands emanate from the notch root. These three plots correspond to the curves marked by "A", "B", and "C" in Fig. 1(b), and to Figs. 5(a)-(c), respectively. Also they are given at the strain levels where the letters "A", "B", and "C $\mathrm{C}$ " are located in Fig. 1(b). It can be observed that with the increase of notch radius, there is a slight decrease of the peak stress in Fig. 1(b), because of the increased strain localization in the shear bands for the condition of large notch radius, e.g., Fig. 5(f). It is also noted that a small degree of numerical fluctuation will break the symmetry. The stress-strain curves in Fig. 1(b) show a decrease of the peak stress with the increase of notch radius, which agrees with the positive 
notch effect. The reduction, however, is not dramatic as the emanated shear bands have already reduced the stress concentration, just like the weak zone model in Section 2. Since the cavitation process is not considered here, the inverse notch effect in Pan et al. [7] will not be predicted.

Effects of the notch size are presented in Fig. 6. From the Rudnicki-Rice model, when the notches are far apart, the horizontal shear bands will not appear as in Fig. 6(a). In other words, shear band patterns in Fig. 4(b) can only be seen for closely spaced notches. For isolated notches or cracks, the Mode-I stress field does not really permit these shear bands to take place. When the notches are closely spaced, the inclined shear bands also interact more intensively, which is also confirmed by the free-volume-based finite element simulations in Fig. 6(f). The three plots in Figs. 6(d)-(f) correspond to the curves marked by "D", "E", and "F" in Fig. 1(b), and to Figs. 6(a)-(c), respectively. Again they are also given at the strain levels where the letters "D", "E", and "F" are located in Fig. 1(b). The peak stress reduces from Fig. 6(d) to Fig. 6(f) because of the increase of notch size. It should be noted that our free-volume model does not have a connection to the shear-localization-induced failure. In other words, the stress-strain curves will extend to infinity in Fig. 1(b). When multiple shear bands occur, e.g., Fig. 6(f), each individual shear band will have a reduced degree of plastic strain so that shear-localization-induced failure will be delayed. That is, geometries like Fig. 6(f) are more desirable in ductility enhancement, although the strength will be reduced for these longer cracks. There are some recent constitutive models where failure criteria are included [26,28,34-36]. For example, Tandaiya et al. [34,35] showed similar shear band patterns and also predicted potential brittle fracture paths based on a Mohr-Coulomb criterion, and Thamburaja [36] predicted the failure when the free volume exceeds a critical value.

The study of notch height effects in Fig. 7 provides a complementary line of information to the inverse-notch-effect study by Pan et al. [7]. With the increase of notch height, or more precisely, the decrease of $\lambda$, Eq. (1) suggests the reduction of $\sigma_{m} / \sigma_{e q}$ and the dominance of shear-localization-induced failure mode. Predictions from the Rudnicki-Rice theory show that a set of shear bands extending from each notch corner into the notched area. Because the angle of the inclined shear bands are about $41^{\circ}$, from a geometric point of view, when $\lambda$ is less than about 1 , these shear bands will run through the entire notched area without any geometric constraint as in Fig. 7(c). The three plots in Figs. 7(d)-(f) are given at the strain levels where the letters " $G$ ", "H", and "I" are located in Fig. 1(b), and also correspond to the conditions in Figs. 7(a)-(c), 
respectively. It can be observed that with the increase of notch height, the shear band patterns change dramatically. Comparing amongst Figs. 7(d)-7(f), it is apparent that the last geometric shape will correspond to a reduced ductility as also shown by the rapid load drop in the stressstrain curve marked with "I" in Fig. 1(b).

Several stress-strain curves in Fig. 1(b) exhibit stress fluctuations post the peak value. A close inspection of the free-volume contour plots suggest that in these cases, multiple shear-band families initiate and propagate at different strain stages until a major family dominates. For example, Fig. 6(d) shows one family of shear bands and the corresponding strain level is before the second stress peak in Fig. 1(b), while Fig. 6(e) shows several shear-band families and the corresponding strain level is after the second stress peak. Such an evolution clearly involves the complex stress-field evolution upon the initiation of shear bands. It should also be noted that the strain in Fig. 1(b) is the ratio of total elongation to the original specimen length, but not the local strain. To this end, these second stress drops correspond to large strain levels such as 0.05 .

Another important observation can be made by comparing to the work in [11], which simulates the shear bands near a single edge notched sample by using a size-dependent constitutive model. Therefore, these authors observed another transition when the geometric size is reduced to nanoscale, i.e., the suppression of shear band. In this case, the deformation rather shows the necking and ligament yielding in the notched are, and the corresponding stress-strain curves do not show steep stress drops. Models presented in our work here do not have a length dependence, and thus the above transition in [11] is not observed. However, it should be noted that the related experiments, e.g., $[1,4,7]$, tested much larger specimens. Furthermore, comparing Fig. 5(f) to Fig. 6(f), it is likely that ligament yielding may take place if the notch length and notch radius are both very large. This is a rather extreme geometric shape not commonly seen in experiments.

\section{Conclusions}

Notched BMGs have been extensively studied in literature. While it is generally believed that a brittle solid will exhibit reduced strength with the introduction of notches, some recent experiments, as complied in Pan et al. [7], found notch insensitivity or inverse notch effects. While this is understood primarily in Pan et al. [7] by the competition between cavitation failure and shear localization, this work adds two important factors that contribute to these notch effects. 
First, when the normal fracture along the notch plane is the dominant mechanism, the largescale-bridging behavior is responsible for the notch insensitivity. In this case, an additional weak zone from the notch roots will further reduce the stress concentration and thus expand the parametric regime for the notch insensitivity. Second, the ductility of notched BMGs depends on how effectively the shear bands are blocked either geometrically or by the decaying stress fields. Explicit predictions of shear band patterns help identify the key roles played by the geometric factors along this line. Lastly, it should be noted that the above two lines are treated rather separately. A comprehensive constitutive model is awaited to integrate the two types of failure modes.

\section{Acknowledgements}

This research was supported by the U.S. Department of Energy, Office of Science, Basic Energy Sciences, Materials Sciences and Engineering Division. 


\section{References}

[1] K.M. Flores, R.H. Dauskardt, Acta Mater. 49 (2001) 2527.

[2] J.X. Zhao, F.F. Wu, R.T. Qu, S.X. Li, Z.F. Zhang, Acta Mater. 58 (2010) 5420.

[3] J.X. Zhao, Z.F. Zhang, Mater. Sci. Eng. A 528 (2011) 2967.

[4] R.T. Qu, M. Calin, J. Eckert, Z.F. Zhang, Scripta Mater. 66 (2012) 733.

[5] B. Sarac, J. Schroers, Nature Comm. 4 (2013) 2158.

[6] X.W. Gu, M. Jafary-Zadeh, D.Z. Chen, Z. Wu, Y.W. Zhang, D.J. Srolovitz, J.R. Greer, Nano Lett. 14 (2014) 5858.

[7] J. Pan, H.F. Zhou, Z.T. Wang, Y. Li, H.J. Gao, J. Mech. Phys. Solids 84 (2015) 85.

[8] Y. Jiang, J. Mater. Res. 31 (2016) 765.

[9] Z.D. Sha, Q.X. Pei, Z.S. Liu, Y.W. Zhang, T.J. Wang, Sci. Rep. 5 (2015) 10797.

[10] Z.D. Sha, Q.X. Pei, V. Sorkin, P.S. Branicio, Y.W. Zhang, H.J. Gao, Appl. Phys. Lett. 103 (2013) 081903.

[11] I. Singh, R. Narasimhan, J. Mech. Phys. Solids 86 (2016) 53.

[12] V. Tvergaard, Adv. Appl. Mech. 27 (1990) 83.

[13] G. Bao, Z. Suo, Appl. Mech. Rev. 45 (1992) 355.

[14] M.Y. He, B. Wu, Z. Suo, Acta Metall. Mater. 42 (1994) 3065.

[15] Y. Liu, Y.F. Gao, J. R. Soc. Interface 12 (2015) 20141042.

[16] Y.F. Gao, L. Wang, H. Bei, T.G. Nieh, Acta Mater. 59 (2011) 4159.

[17] Z.N. An, W.D. Li, F.X. Liu, P.K. Liaw, Y.F. Gao, Metall. Mater. Trans. A 43 (2012) 2729.

[18] H.L. Jia, F.X. Liu, Z.N. An, W.D. Li, G.Y. Wang, J.P. Chu, J.S.C. Jang, Y.F. Gao, P.K. Liaw, Thin Solid Films 561 (2014) 2.

[19] I. Singh, T.F. Guo, P. Murali, R. Narasimhan, Y.W. Zhang, H.J. Gao, J. Mech. Phys. Solids 61 (2013) 1047.

[20] I. Singh, T.F. Guo, R. Narasimhan, Y.W. Zhang, Int. J. Solids Struct. 51 (2014) 4373.

[21] Y. Chen, L.H. Dai, Int. J. Plast. 77 (2016) 54.

[22] Y.F. Gao, A.F. Bower, Modelling Simul. Mater. Sci. Eng. 12 (2004) 453.

[23] V. Tvergaard, J.W. Hutchinson, J. Mech. Phys. Solids 40 (1992) 1377.

[24] L.L. Zheng, Y.F. Gao, S.Y. Lee, R.I. Barabash, J.H. Lee, P.K. Liaw, J. Mech. Phys. Solids 59 (2011) 2307.

[25] Y.F. Gao, Extreme Mech. Lett. 6 (2016) 45.

[26] Y. Chen, M.Q. Jiang, Y.J. Wei, L.H. Dai, Phil. Mag. 91 (2011) 4536.

[27] P. Murali, R. Narasimhan, T. Guo, Y.W. Zhang, H. Gao, Scripta Mater. 68 (2013) 567.

[28] M.Q. Jiang, G. Wilde, J.H. Chen, C.B. Qu, S.Y. Fu, F. Jiang, L.H. Dai, Acta Mater. 77 (2014) 248.

[29] K.M. Flores, R.H. Dauskardt, J. Mater. Res. 14 (1999) 638.

[30] P. Tandaiya, R. Narasimhan, U. Ramamurty, Acta Mater. 61 (2013) 1558.

[31] J.W. Rudnicki, J.R. Rice, J. Mech. Phys. Solids 23 (1975) 371.

[32] F. Spaepen, Acta Metall. 25 (1977) 407.

[33] Y.F. Gao, Modelling Simul. Mater. Sci. Eng. 14 (2006) 1329.

[34] P. Tandaiya, R. Narasimhan, U. Ramamurty, Acta Mater. 55 (2007) 6541.

[35] P. Tandaiya, U. Ramamurty, R. Narasimhan, J. Mech. Phys. Solids 57 (2009) 1880.

[36] P. Thamburaja, J. Mech. Phys. Solids 59 (2011) 1552. 


\section{Figure Captions}

Figure 1 (a) Geometric illustration of the double-edge notched sample, from which the stress state is given in Eq. (1). (b) The engineering stress-strain curves from the free-volume-based finite element simulations, corresponding to the results in Figs. 5-7. The loading and boundary conditions used in the finite element simulations are also prescribed. The nine curves marked by "A"-"I" have different notch radius $r$, length $a$, and height $w$, as given in the legend. Also these letters are placed at certain strain levels that correspond to the contour plots in Figs. 5-7.

Figure 2 (a) Model setup for the study of the synergistic effects of the cohesive fracture along the notched area and the weak zone that represents the shear band emanating from the notch root. (b) The normal traction-separation law used in the cohesive elements in (a). (c) The tangential traction-separation law used to represent the weak zone in (a). The extent of the weak zone is calculated from the boundary value problem.

Figure 3 The fracture stress, $\sigma_{c r t}$, is governed by the ratio of the cracked length and the cohesive zone size, i.e., $a \sigma_{0} / E \delta_{0}$. The notch-insensitivity regime is approached when $a$ is comparable to or less than $\sigma_{0} / E \delta_{0}$, also corresponding to the largescale-bridging (LSB) behavior. The LSB regime will be expanded if the weak zone has a low strength or a low stiffness, compared to the cohesive crack along the notched area.

Figure 4 Three commonly observed shear band configurations in double-edge notched BMGs subjected to uniaxial tensile loading: (a) radial shear bands [2,3], (b) curved shear bands that connect the two notches [4,5], and single inclined shear bands that run through the notched area [7].

Figure 5 Effects of the notch tip radius, $r$, on the shear band patterns. (a)-(c) Predictions from the Rudnicki-Rice instability analysis. (d)-(e) Free-volume-based finite element simulations, which are snapshots taken from the deformation states at "A", "B", "C" in Fig. 1(b). The contour plots are for the normalized free volume, $v_{f} / \alpha v^{*}$. 
Figure 6 Effects of the notch length, $a$, on the shear band patterns. (a)-(c) Predictions from the Rudnicki-Rice instability analysis. (d)-(e) Free-volume-based finite element simulations, which are snapshots taken from the deformation states at "D", "E", "F" in Fig. 1(b).

Figure 7 Effects of the notch height, $w$, on the shear band patterns. (a)-(c) Predictions from the Rudnicki-Rice instability analysis. (d)-(e) Free-volume-based finite element simulations, which are snapshots taken from the deformation states at " $\mathrm{G}$ ", " $\mathrm{H}$ ", "I" in Fig. 1(b). 

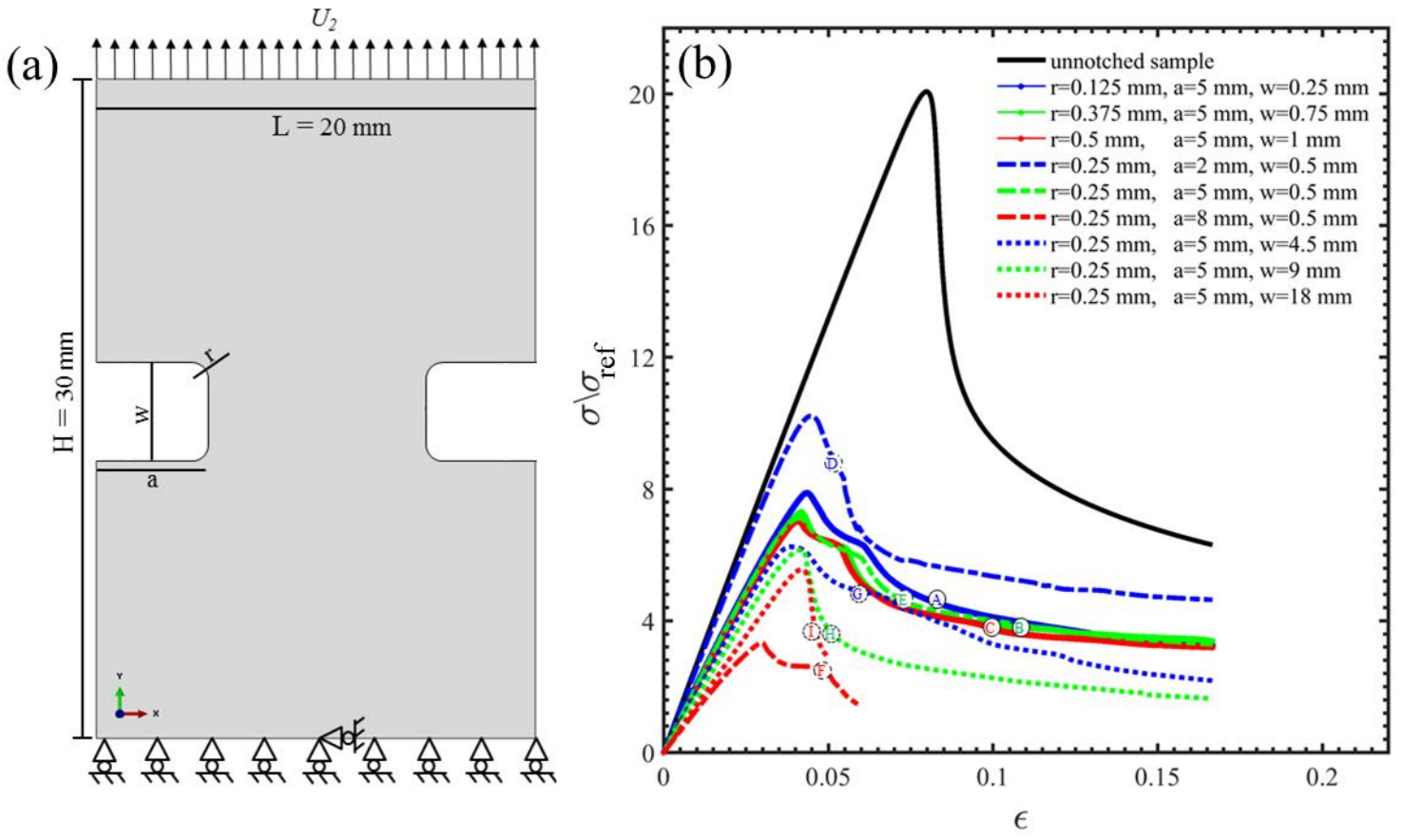

Figure 1 

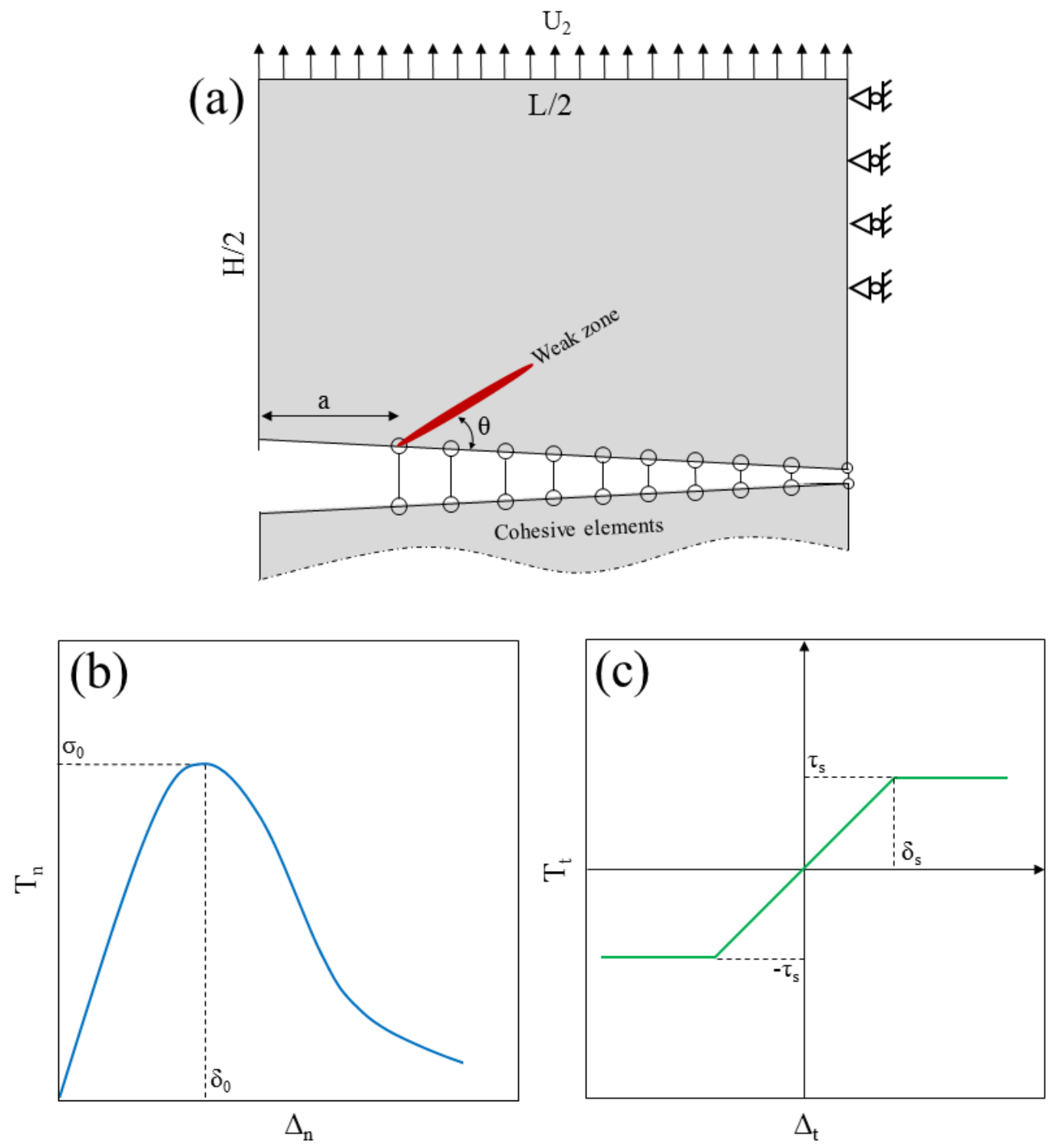

Figure 2 


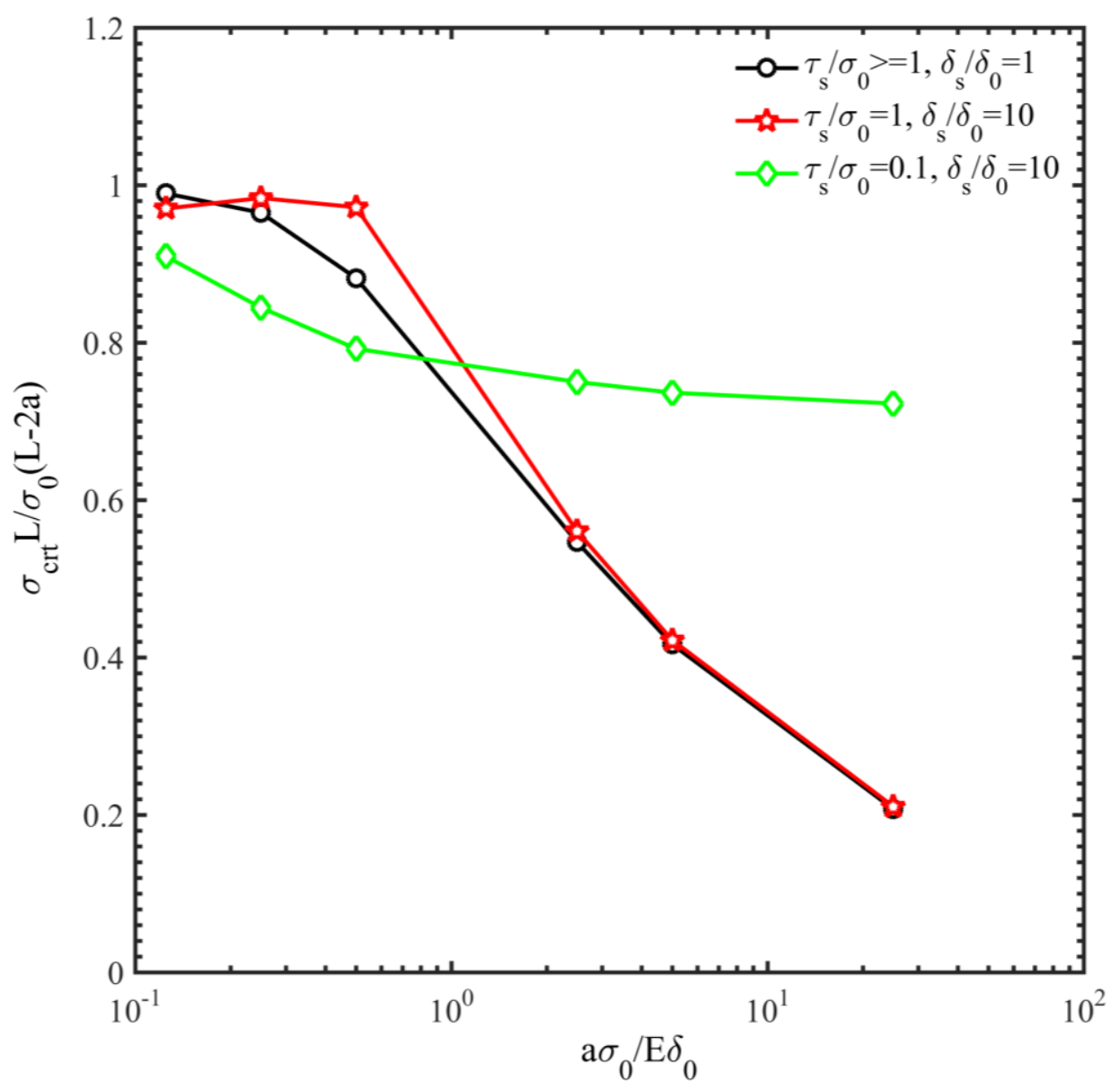

Figure 3 

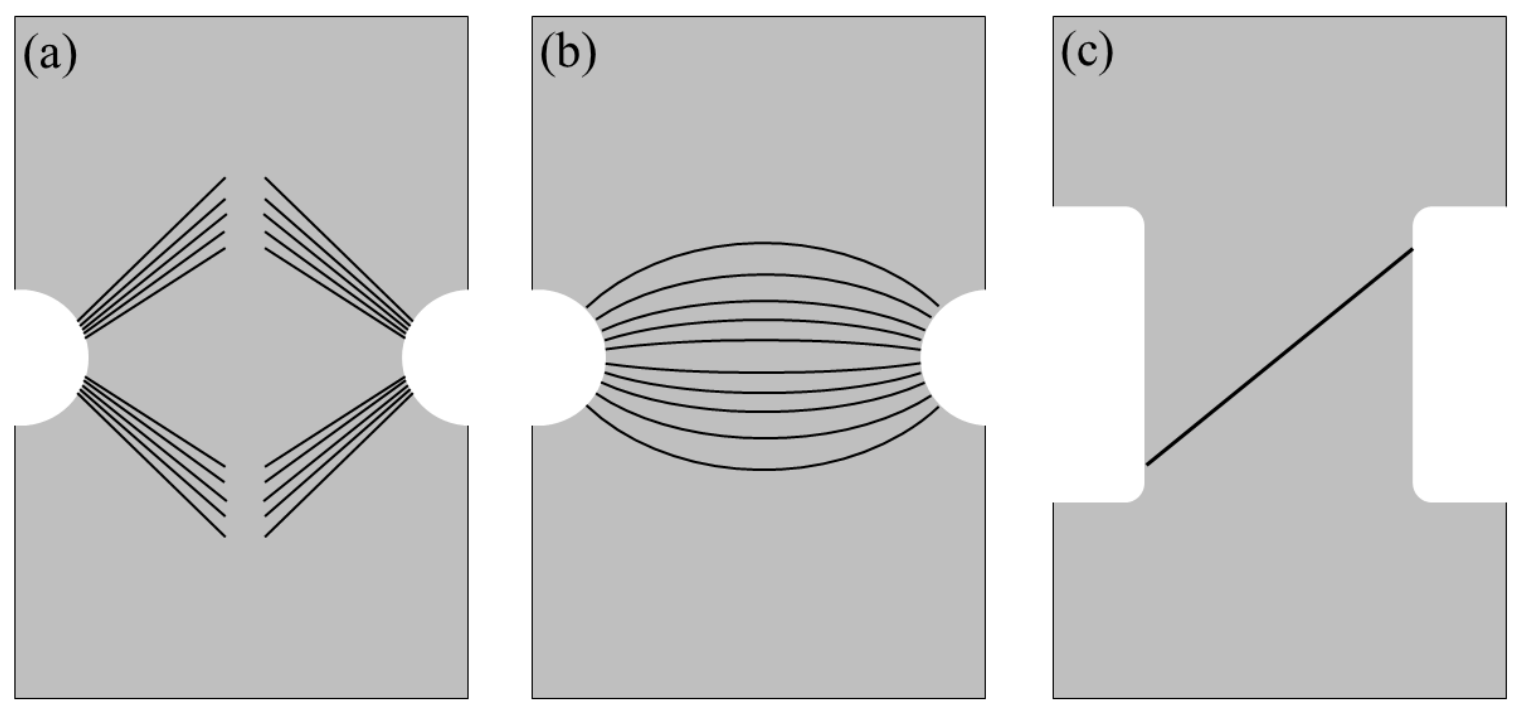

Figure 4 

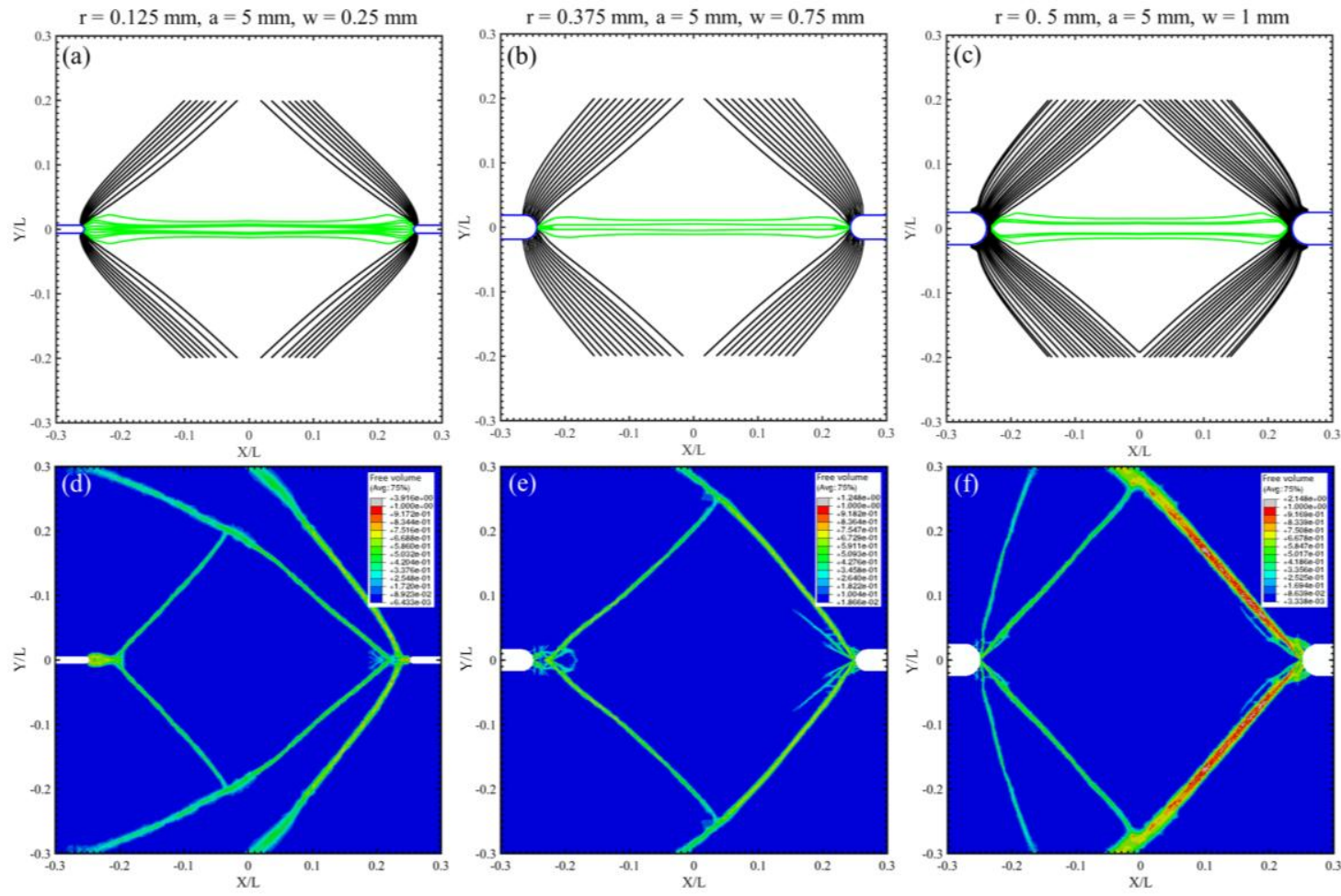

Figure 5 

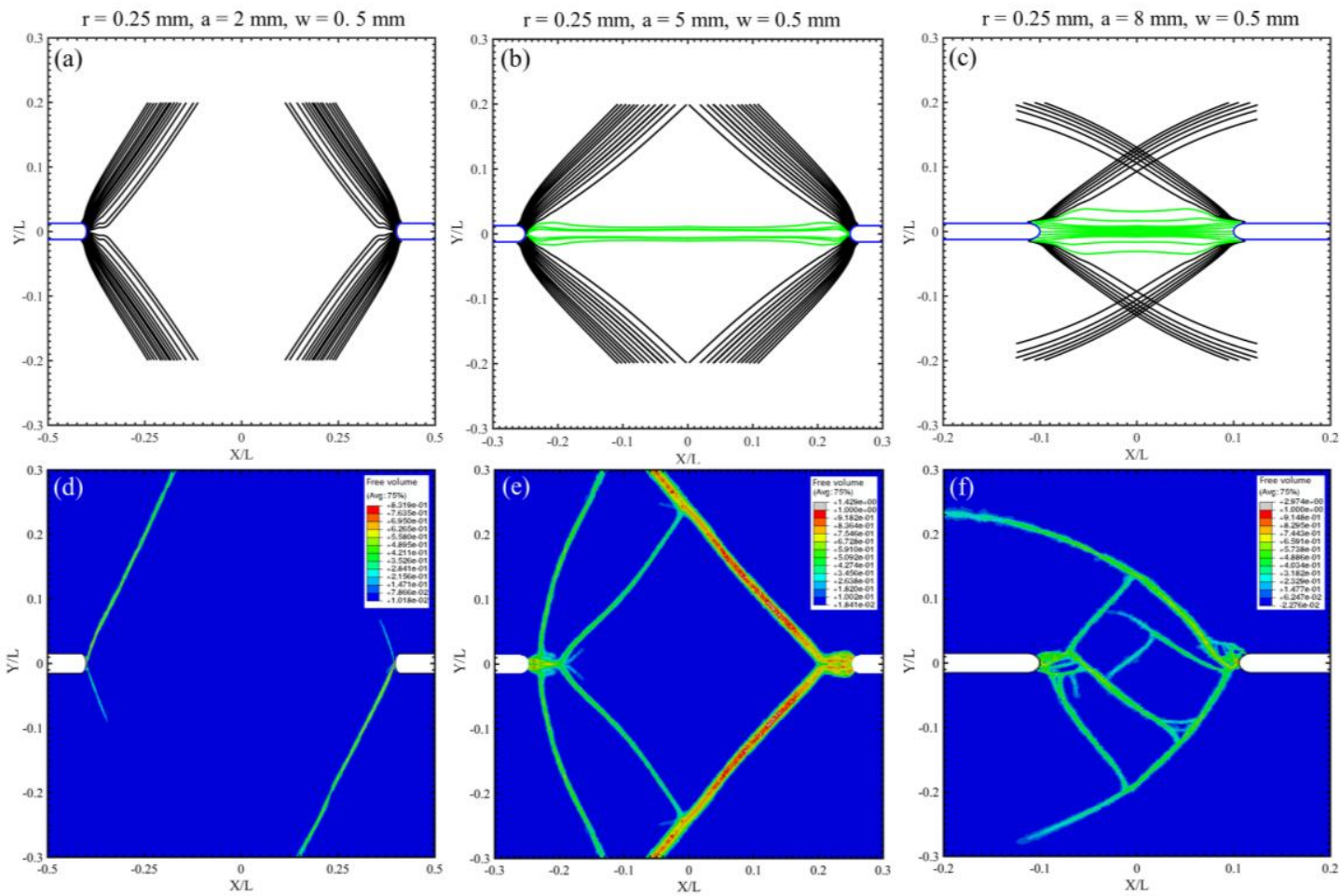

Figure 6 

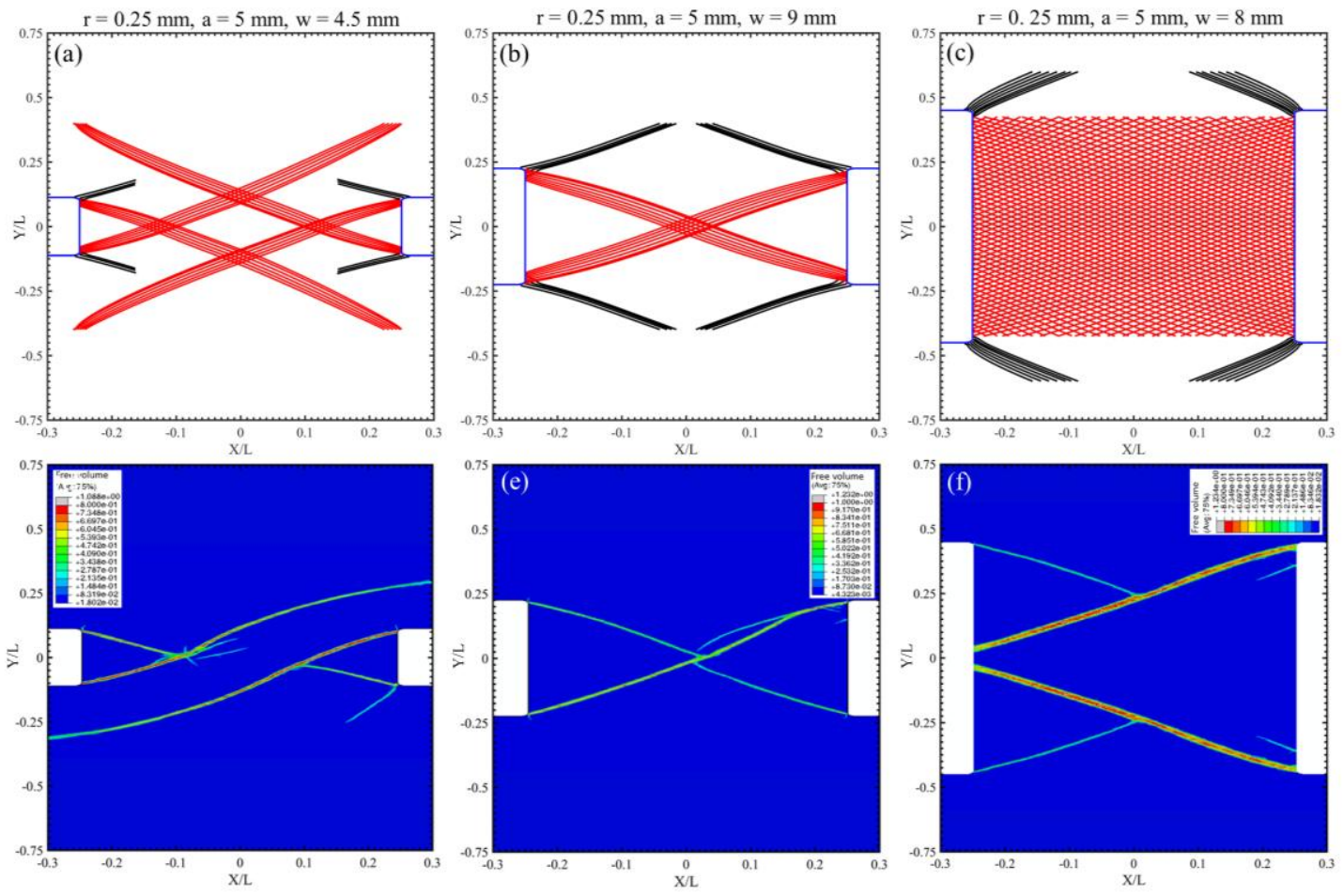

Figure 7 